\title{
Arbeitsgruppe Handosteosynthesen der DAOI
}

प H. R. Siebert

\section{Zusammenfassung \\ Ziele der Arbeitsgruppe Hand- osteosynthesen sind: \\ - Fortbildung im Bereich Hand- traumatologie durch: \\ - spezielle Handtraumakurse \\ - Workshops \\ - intensive Beschäftigung mit Be- fund-Dokumentation und deren Bewertung (Score) \\ - Beteiligung an der klinischen Überprüfung von neuen Implan- taten und Instrumenten der AO (z.B. 3,0 mm kanülierte Schrau- be Scaphoid, Kompakt-Set) \\ - Klärung klinischer Fragestellun- gen durch multizentrische Stu- dien (Mittelhand-Studie 1998 bis 2000) \\ - internationaler Austausch durch gemeinsame Kurse und klinische Studien, Hospitationen}

\section{Einleitung}

Die Arbeitsgruppe Handosteosynthesen wurde vor 10 Jahren eingerichtet, um der zunehmenden Spezialisierung im Bereich der Implantate, Instrumente und Indikationsstellung der AO Rechnung zu tragen. Dabei sollten in einer Arbeitsgruppe alle an der Hand und seiner Verletzung interessierten AO-Mitglieder, aber auch einzelne Experten die Möglichkeit haben, Implantate zu überprüfen, neue mit zu entwickeln oder bei klinischen Überprüfungen Erfahrungen zu sammeln.

OP-JOURNAL 2000; 16: 282-284

(C) Georg Thieme Verlag Stuttgart · New York

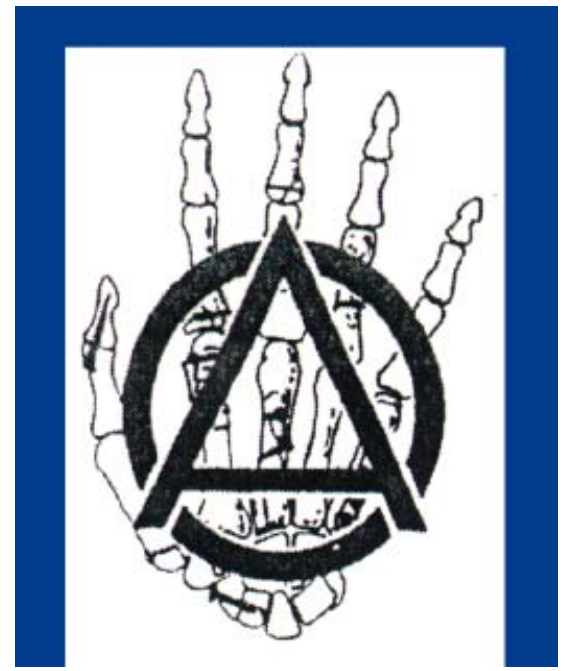

Abb.1 Emblem der Arbeitsgruppe Handosteosynthese der DAOI.

\section{Zur Geschichte der Klein- und Mini-Implantate der AO:}

In den frühen 60er Jahren setzte die Entwicklung kleiner Implantate und die standardisierte Anwendung im Bereich der kleinen Skelettanteile durch die Arbeiten von Heim [1] und Pannike [2] ein. Durch die damals vorhandene Technik war man erstmals in der Lage, mit relativ kleinen Implantaten instabile Verletzungen des Fuß- und Handskeletts durch übungsstabile Osteosynthesen zu versor- gen und damit eine Idee der Arbeitsgemeinschaft für Osteosynthesefragen (frühfunktionelle gipsfreie Nachbehandlung) auch im Bereich kleiner Skelettanteile umzusetzen (Abb.2). Die klinischen Erfolge dieser beiden Pioniere überzeugten, so dass die Entwicklung noch kleinerer Implantate $(2,7$ bis 2,0$)$ in den 70 er Jahren möglich wurde (Abb.3). Schon damals wurde die Bedeutung einer systematischen Fortbildung in Form von speziellen Handkursen erkannt und diese in der Schweiz, Österreich und Deutschland durchgeführt.

In den 80er Jahren wurde die Vielfalt der unterschiedlichen Implantatgrößen für die Hand in einem kompakten Set (Abb.4) bis auf die Dimension von $1,1 \mathrm{~mm}$ weiter entwickelt, erstmalig wurden selbstschneidende Schrauben probeweise entwickelt und kanülierte Schrauben als Zugschrauben für die Behandlung von z. B. Carpalfrakturen als Prototyp vorgestellt. Dabei mussten die Hersteller der AO Implantate auf die bereits vielfach klinisch erprobten und bewährten „MiniImplantate“ anderer Firmen reagieren. Die Systematisierung der Implantate und Instrumente wie auch der Einsatz von Titan anstelle von Stahl führte zu einer weiten Verbreitung der Implantate, jedoch blieb Indikationsstellung und Technik vielen Anwendern verborgen, so dass aufgrund von Misserfolgen und

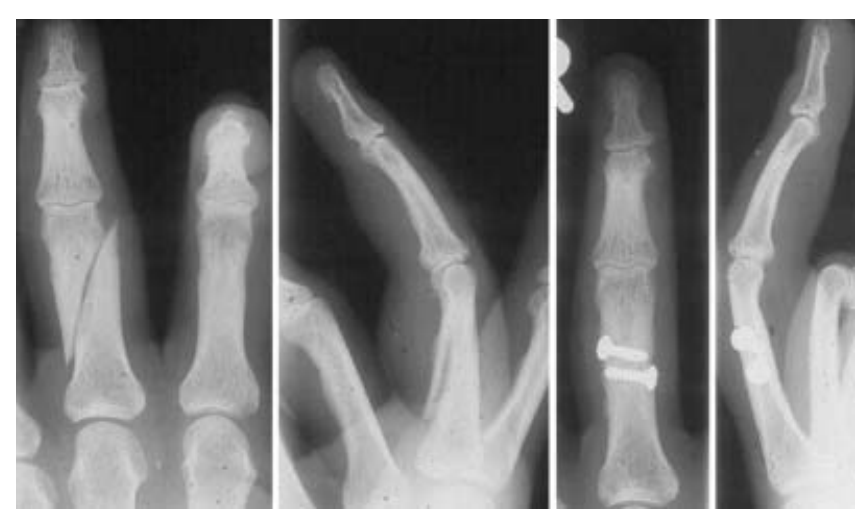

Abb. 2 Lange, instabile Schrägfraktur Ph 1: ZugschraubenOsteosynthese 2,0 mm Stahl 1974 

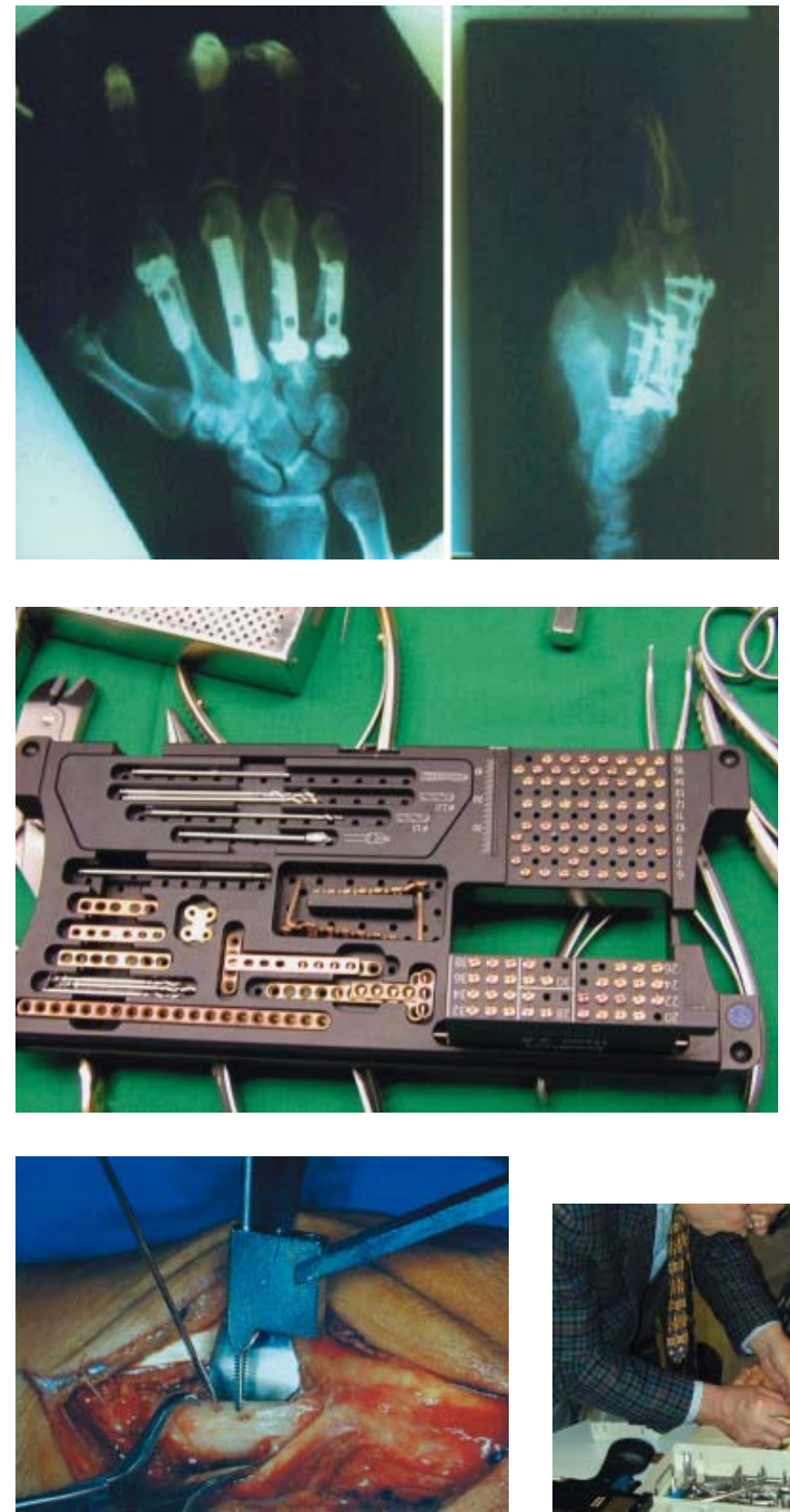

Abb.5 Intraoperativer Situs: MC II-Fraktur. Abbildung aus Lehrbuch. Obsolete Technik, da exzessive Knochenfreilegung!

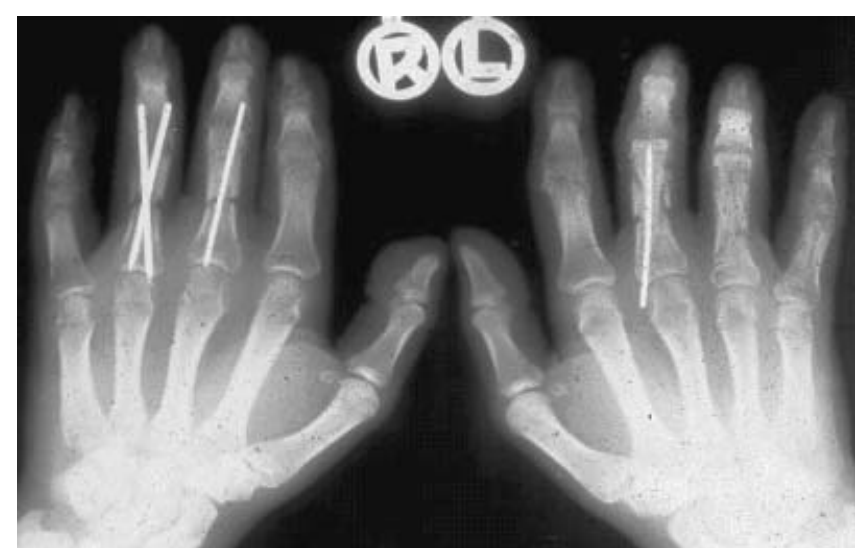

Abb. 4 KompaktHand-Set: Dimension 2,0 mm (AOI-Entwicklung 1987 1989).

Abb. 3 Instabile Serienfrakturen Metacarpalia II bis V: Verschiedene $2,0 \mathrm{~mm}$ Titan-Platten 1984.

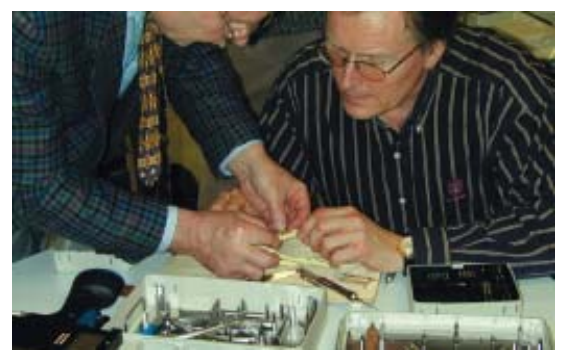

Abb. 7 AO Handkurs: Übung am Kunststoffknochen-Modell. 1993.

Abb. 6 KirschnerDraht-Osteosynthese bei Schaftquerfrakturen Ph 1 D 3 und D 4. rechts: Falsche Technik!
Fehlleistungen die Notwendigkeit erwuchs, diese hervorragende Technik systematisch auch den wenig Geübten mitzuteilen (Abb.5 u. 6). Petracic war der Erste in Deutschland, der diese Entwicklung voraussah und zielstrebig eigene Handkurse in Oberhausen, erstmalig auch an Leichenpräparaten abhielt. Er entwickelte in Davos Lehr-Videos für die unterschiedlichsten Indikationsstellungen und Techniken und wurde logischerweise 1. Leiter des Arbeitskreises Handosteosynthesen. Zeitlich etwas versetzt wurde vor allem in der Schweiz (Oberndorf, Lowka) und Österreich (Wiener-Handkurse Böhler und Grazer Handkurse Syskowicz) die Unterrichtung in den speziellen handtraumatologischen Techniken aufgegriffen und diese Kurse bis zur Perfektion entwickelt, so dass sie einen nicht wegzudenkenden Stellenwert im Fortbildungswesen der Handtraumatologie haben. Im Osten Deutschlands wurden in der sehr engagierten Handchirurgie der Universität Jena von Friedel ebenfalls AO-Handkurse eingerichtet, die noch heute ihre große Anziehungskraft haben (Abb. 7).

\section{Die Arbeit des Arbeitskreises seit 1996}

Seit 1995 hat sich ein größerer Kreis von handtraumatologisch interessierten Ärztinnen und Ärzten aus AO-Kliniken wie aus handchirurgischen Zentren getroffen, um folgende Themenkreise zu bearbeiten:

a) Entwicklung einer alpha-numerischen Klassifikation von Verletzungen des Handskeletts (Petracic, Siebert 1993) (Abb. 8).

b) Entwicklung eines allgemein einsetzbaren Befund- und Dokumentationssystems, differenzierter Nachuntersuchung und EDV-gestützter Dokumentation sowie Aufstellung von Bewertungsmaßstäben (EDV-gestützte Dokumentation: Germann)

c) Hand-on-Workshops für die Mitglieder des Arbeitskreises: Jährlich wechselnd in unterschiedlichen Mitglieder-Kliniken, bisherige Themen:

- Fixateur externe-Typen an der Hand (Werber, München)

- Verschraubung Scaphoid-Fraktur verschiedene Implantate, Carpale Bandverletzungen (Laier, Karlsruhe) (Abb.9)

- Handgelenkarthroskopie und Weichteildefektdeckung an der Hand (Helling, Köln).

Bei diesen Workshops mit einer Teilnehmerzahl von 20 bis 40 wurden intensiv 

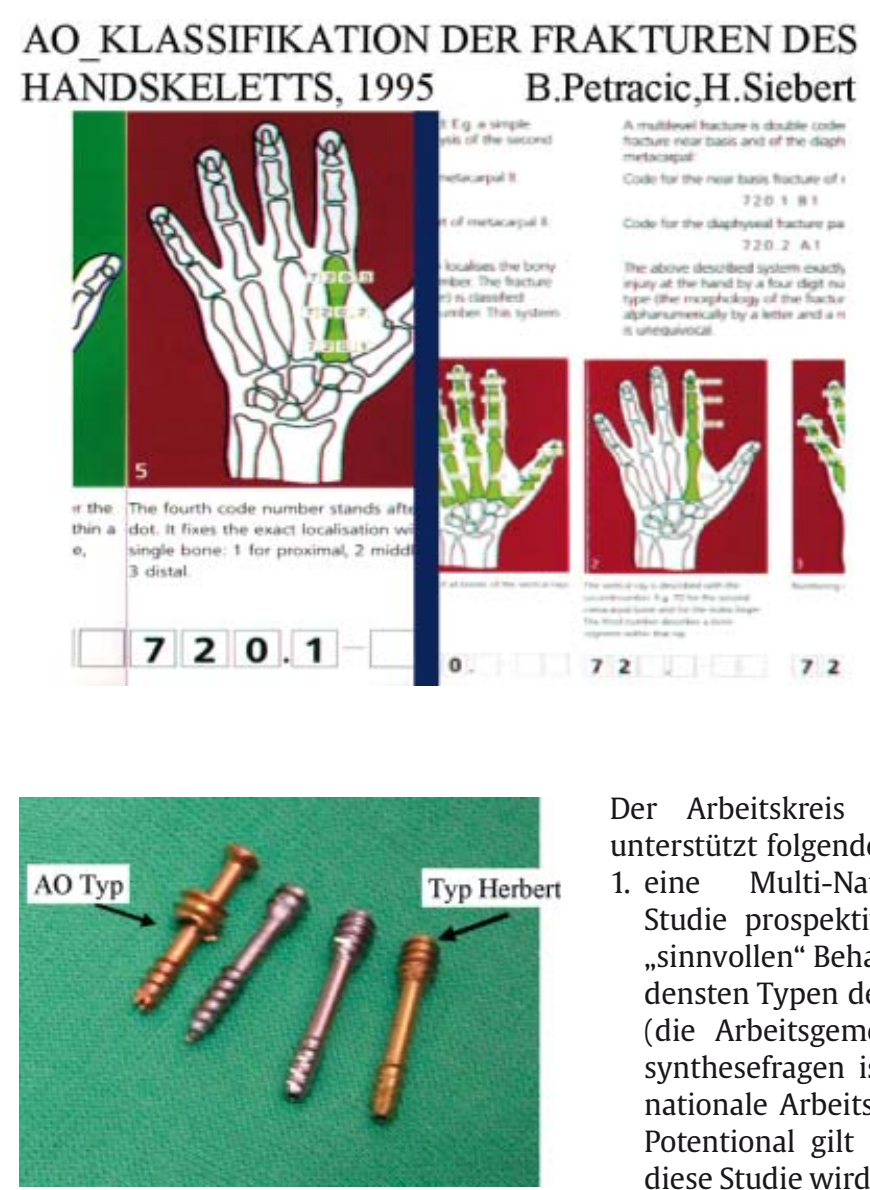

Abb. 9 Kanülierte Zugschrauben verschiedener Hersteller.

die jeweiligen Themen theoretisch und praktisch erarbeitet und dabei Implantate der verschiedensten Hersteller verglichen.

d) Prospektive Multi-Zenter-Studie zur Mittelhandfraktur: Mit einem erheblichen personellen und vor allem logistisch finanziellen Aufwand konnten Dank großzügiger finanzieller Unterstützung durch die AO-International insgesamt ca. 1500 Mittelhandfrakturen erfasst, deren Behandlung dokumentiert und die Ergebnisse von ca. 800 nachuntersuchten Patienten nach 6 bis 12 Monaten ausgewertet werden. Neben interessanten epidemiologischen Ergebnissen kann festgestellt werden, dass auch scheinbar stabile Frakturen häufig operativ versorgt werden und damit eine gewisse „Überbehandlung“ festzustellen ist. Diese über 3 Jahre gehende Studie hat zu einer engen Vernetzung zwischen den beteiligten Einrichtungen und Zentren geführt, so dass sich daraus eine teilweise enge klinische $\mathrm{Zu}$ sammenarbeit im Sinne von Tele-Consulting entwickelt hat.
Abb. 8 Ausschnitt aus AO-Klassifikation der Frakturen des Handskeletts.
Der Arbeitskreis Handosteosynthesen unterstützt folgende zukünftige Projekte 1. eine Multi-Nationale-Multi-ZenterStudie prospektiv zur Erfassung der „sinnvollen“ Behandlung der verschiedensten Typen der Kahnbeinfrakturen (die Arbeitsgemeinschaft für Osteosynthesefragen ist primär eine internationale Arbeitsgemeinschaft, dieses Potentional gilt es zu nutzen). Auch diese Studie wird nur durch eine großzügige Unterstützung durch das neu geschaffene Department für Clinical Investigation and Documentation der AO-International in Davos möglich sein.

2. Es gilt winkelstabile Implantate der Dimension 2,0 und 1,5 als Ersatz der Mini-Condylenplatte zu entwickeln und klinisch zu erproben (TK: Rüdigier, Offenburg)

3. Sowohl die kanülierte Scaphoidschraube mit gewindetragender Unterlegscheibe als auch die neuen Spezialplatten für die Behandlung von intraartikulären distalen Radiusfrakturen bedürfen der Modifikation aufgrund der vielfach gewonnenen Erfahrungen im klinischen Gebrauch.

4. In Deutschland will die Arbeitsgruppe sich noch mehr aktiv in das Kurswesen der DAOI einbringen. Insbesondere wird es als wünschenswert angesehen, spezielle Hand-Trauma-Kurse - für Anfänger und Fortgeschrittene - in den verschiedenen Regionen unserer Republik unter Nutzung der bereits bestehenden Einrichtungen weiter zu entwickeln.

\section{Schlußfolgerung}

Nach den Pioniertaten Einzelner, die Philosophie und Technik der operativen Frakturenbehandlung an den großen Röhrenknochen auf den Bereich der Hand umzusetzen, wurde ein Arbeitskreis der Deutschen Sektion der Arbeitsgemeinschaft für Osteosynthesefragen geschaffen, der sich speziell mit den Fragestellungen der operativen aber auch funktionell konservativen Behandlung von Verletzungen der Hand beschäftigt und dabei:

- die bisher zur Verfügung stehenden Implantate, Techniken und Indikationen kritisch untersucht,

n durch enge partnerschaftliche Zusammenarbeit in Studien und Workshops neue Entwicklungen fördert,

- im internationalen Austausch jüngeren Mitarbeitern Hospitationen zu ermöglichen.

Nur der ständige und kritische Dialog in vertrauter Umgebung ohne all zu großem Wettbewerbsklima ist der ideale Soziotop für eine erfolgreiche Arbeit. Diesen Raum bietet ein Arbeitskreis wie der der Deutschen Sektion der AO International im Rahmen der Handosteosynthesen, wo nicht Titel und Dienstalter, sondern Engagement und Ideen gefragt sind. Wir werden weiterhin Bestehendes in Frage stellen, Neues aufgreifen und jede/n willkommen heißen, der/die Interesse hat, kontinuierlich auf dem Gebiet der Handosteosynthesen sich fortzubilden und Entwicklungen anzustoßen.

\section{Literatur}

${ }^{1}$ Heim U, Pfeiffer KM. Periphere Osteosynthesen Springer Verlag 1. Auflage 1972

2 Pannnike A. Osteosynthesen in der Handchirurgie Springer Verlag Berlin Heidelberg 1972

3 Petracic B, Siebert HR. AO-Klassifikation der Frakturen des Handskeletts Akt. Traumatologie 1995; 25: $163-166$

Prof. Dr. med. H. R. Siebert Chefarzt

Chirurgische Klinik II

Unfall-, Hand-, Wiederherstellungschirurgie

Diakonie-Krankenhaus

Diakoniestr. 10

74523 Schwäbisch Hall 